\title{
F Sonatem \\ Identification of Candidate Biomarkers Correlated Poorer Prognosis of Breast Cancer via Bioinformatics Method
}

\section{Gang Chen}

Department of Breast Surgery, The Affiliated Yantai Yuhuangding Hospital of Qingdao University

\section{Mingwei Yu}

Department of Orthopedics, The Affiliated Yantai Yuhuangding Hospital of Qingdao University

\section{Jianqiao Cao}

Department of Breast Surgery, The Affiliated Yantai Yuhuangding Hospital of Qingdao University

\section{Huishan Zhao}

Reproductive Medicine Centre, The Affiliated Yantai Yuhuangding Hospital of Qingdao University

\section{Yuanping Dai}

Department of Medical Genetics, Liuzhou Maternal and Child Health Hospital

\section{Yizi Cong}

Department of Breast Surgery, The Affiliated Yantai Yuhuangding Hospital of Qingdao University Guangdong Qiao ( $\nabla$ qiaogddxy@163.com )

Department of Breast Surgery, The Affiliated Yantai Yuhuangding Hospital of Qingdao University

\section{Research Article}

Keywords: Bioinformatics, Breast cancer, Differential expressed genes, Gene Expression Omnibus

Posted Date: April 16th, 2021

DOl: https://doi.org/10.21203/rs.3.rs-402557/v1

License: (9) This work is licensed under a Creative Commons Attribution 4.0 International License. Read Full License 


\section{Abstract}

Background: Breast cancer (BC) is a malignancy with a high incidence among women in the world, and it is very urgent to identify significant biomarkers and molecular therapy methods.

Methods: Total 58 normal tissues and 203 cancer tissues were collected from three Gene Expression Omnibus (GEO) gene expression profiles, and the differential expressed genes (DEGs) were identified. Subsequently, the Gene Ontology (GO) function and Kyoto Encyclopedia of Genes and Genome (KEGG) pathway were analyzed. Additionally, hub genes were screened by constructing a protein-protein interaction (PPI) network. Then, we explored the prognostic values and molecular mechanism of these hub genes Kaplan-Meier (KM) curve and Gene Set Enrichment Analysis (GSEA).

Results: 42 up-regulated and 82 down-regulated DEGs were screened out from GEO datasets. GO and KEGG pathway analysis revealed that DEGs were mainly related to cell cycles and cell proliferation. Furthermore, 12 hub genes (FN1, AURKA, CCNB1, BUB1B, PRC1, TPX2, NUSAP1, TOP2A, KIF20A, KIF2C, RRM2, ASPM) with a high degree of genes were selected, among which, 11 hub gene were significantly correlated with the prognosis of patients with BC. From GSEA reviewed correlated with KEGG_CELL_CYCLE and HALLMARK_P53_PATHWAY.

Conclusion: this study identified 11 key genes as BC potential prognosis biomarkers on the basis of integrated bioinformatics analysis. This finding will improve our knowledge of the $\mathrm{BC}$ progress and mechanisms.

\section{Background}

Breast cancer is the most common malignant tumor among women in the world [1], which also is the leading cause of woman death [2]. Although many methods for BC early diagnosis, actually, effectively approaching still limited. Currently, the normal treatment of BC mainly includes surgery, chemotherapy and radiotherapy or endocrine therapy et al, but patients with a 5-year survival rate are still very low [3], so it is very urgent to identify significant biomarkers and molecular therapy methods [4].

Previous studies screened the biomarkers predictors and function enrichment, mostly using online tools, such as GEO2R, DAVID or KOBAS et al. Actually, the majority of the online database are not precise enough, due to the slow update. Besides, the online DE analysis tool GEO2R seems not to normalize original data, so there might be a great deviation for each probe.

In this study, we identified several key genes that could be used as a sensitivity biomarkers for the diagnosis of BC using the GEO database. We downloaded three different region source gene expression profiles (GSE29044, GSE42568, GSE50428) from the GEO database, and a total of 261 breast tissue samples were included in this study, consisting of 58 normal breast tissue samples and 203 BC tissue samples. Then, the limma package of $\mathrm{R}$ software and Venn diagram online tool were applied to DEGs in the three datasets above. Furthermore, GO function and KEGG pathway analysis were conducted by the R 
software, and the newest annotation files were downloaded from the official website respectively. We constructed a protein and protein interaction (PPI) network with Cytoscape and the hub genes were screened by the cytoHubba plugin. For the validation of the DEGs, the BC section in the TCGA database consisting of $1102 \mathrm{BC}$ tissues and 113 normal tissues was downloaded using TCGA-Assembler [5] package of $R$ software, and those DEGs from GEO were verified by the TCGA database. Finally, only 11 genes were selected as BC potential prognosis biomarkers by KM-plot, and GSEA analyses were involved to study the potential molecular mechanisms of these hub genes. In conclusion, our study provides some potential sensitive biomarkers for $\mathrm{BC}$ patients and promotes an understanding of the molecular mechanisms of $\mathrm{BC}$ progression.

\section{Methods}

\section{Microarray data information}

The GEO (http://www.ncbi.nlm.nih.gov/geo/) database is a free public database of microarrays and is used for gene expression datasets and platform records [6]. The gene expression profiles of GSE29044, GSE42568, GSE50428 were chosen from the GEO database. GSE29044 was based on the GPL570 platform containing 36 normal breast tissues and 73 BC tissues. GSE42568 was based on the GPL570 platform containing 17 normal breast tissues and 104 BC tissues. GSE50428 was based on the GPL13648 platform containing 5 normal breast tissues and 26 BC tissues. The downloaded data was tidied using the Perl (Practical Extraction and Report Language, Version 5.30.2) software, then log2 transformation and Z score standardization were performed on all data of gene expression.

\section{Screening for DEGs}

DEGs between BC samples and normal breast samples were identified using the limma (version 3.30.0) package of $\mathrm{R}$ (version 3.5.1) software, which compared two groups of samples in most GEO series to obtain genes with different expressions under the same experimental conditions. The DEGs with $\mathrm{FC} \geq 1.5$ or $\mathrm{FC} \leq 1 / 1.5$ and adjust $\mathrm{P}<0.05$ were considered as the cutoff criteria. Then, we used Venn software online (http://bioinformatics.psb.ugent.be/webtools/Venn/) to obtain the common DEGs in all three independent cohorts.

\section{GO term and KEGG pathway enrichment analysis of DEGs}

The GO annotation has given us a conspicuous meaningful and for the variety of biological functional from microarray and other big datasets [7]. KEGG is a systematic gene and genomic function information database, the gene functional information is stored in the PATHWAY segments [8]. GO and KEGG annotation was downloaded from the official website respectively (http://current.geneontology.org/products/pages/downloads.html, https://www.genome.jp/keggbin/get_htext?hsa00001+3101, 2021-01-05download), and enrichment of DEGs was performed using hypergeometric distribution formula of $\mathrm{R}$ software, we regarded P-value $\leq 0.05$ with fold change more than 2 as a statistically significant difference and significant enrichment. 


\section{PPI network construction, hub gene identification}

After enrichment of the DEGs, we construct the PPI network based on String online database (https://string-db.org/) for all DEGs [9] and Combine score greater than 0.9 as the cutoff criterion, and then the network was loaded to the Cytoscape (version 3.7.0) software, and cytoHubba plugin was curried to predict hub genes [10].

\section{Validation of the hub genes in the TCGA database}

The TCGA_BRCA dataset was downloaded using the TCGA-Assembler (version 2.0.6) package of $R$ software and then the up and down-regulated hub gene expression levels were verified by the DESeq package of $\mathrm{R}$ software. Then the potential function of the hub genes was analyzed by the GSEA (version 4.1.0).

\section{Survival analysis}

To further investigate the value of hub genes in breast cancer patients, The Kaplan-Meier plotter (http://kmplot.com/analysis/) analysis was conducted [11]. BC database was applied to estimate the prognosis values of hub genes we had identified, each gene was separated into two parts according to the median of expression levels, and if the $p$-value $\leq 0.05$, it would be considered statistically significant.

\section{Statistical analysis}

All statistical analyses were performed using $R$ software, most data were expressed as the mean \pm standard deviation (SD) from the dataset. Statistical analyses were conducted two-tailed and none paired Student's t-test by the GraphPad Prism 5(GraphPad Inc., San Diego, CA, USA) software, and $p \leq 0.05$ were considered as statistical significance [12].

\section{Results}

\section{Identification of DEGs in BC}

A total of 182, 705, 681 up-regulated and 351, 914, 944 down-regulated genes were filtered from GSE29044, GSE42568, and GSE50428 respectively ( $F C \geq 1.5$ or $F C \leq 1 / 1.5$ and adjust $P<0.05$ ). The volcano plot and heat map of DEGs were shown (Figure1. A-C, F-H). In addition, 41 and 86 overlapped up and down-regulated DEGs were screened out by online Venn software from three gene expression profiles (Figure1. D, E).

\section{GO functional enrichment analysis of DEGs}

For the up-regulated DEGs, BP terms are most significantly enriched in cell division, negative regulation of $B$ cell differentiation, and anaphase-promoting complex-dependent catabolic process. CC terms are most significantly enriched in spindle, collagen-containing extracellular matrix, and kinetochores. As for the MF, extracellular matrix structural constituent, microtubule binding, and microtubule motor activity are mostly 
enriched (Figure2. A-C). Besides, for the down-regulated DEGs, BP terms are most significantly enriched in the cellular response to heparin, retinol metabolic process, positive regulation of fat cell differentiation. $\mathrm{CC}$ terms are most significantly enriched in the extracellular space, collagen-containing extracellular matrix, and extracellular region. As for the MF, heparin binding, retinal dehydrogenase activity, and DNAbinding transcription activator activity, RNA polymerase II-specific are mostly enriched (Figure2. E-G).

\section{KEGG pathway enrichment analysis of DEGs}

The method of enrichment is as same as that of the GO enrichment analysis. The up-regulated DEGs were most significantly enriched in the p53 signaling pathway, progesterone-mediated oocyte maturation, protein digestion and absorption. As for the down-regulated DGEs, they are mainly enriched in the AMPK signaling pathway, Adipocytokine signaling pathway, and PPAR signaling pathway (Figure2. D, H).

\section{PPI network construction and hub gene screen}

The degree of the per node was calculated by the Cytohubba plugin囚and the top 5 degrees of DEGs are considered as the hub genes of BC, 12 up-regulated (FN1, AURKA, CCNB1, BUB1B, PRC1, TPX2, NUSAP1, TOP2A, KIF20A, KIF2C, RRM2, ASPM) and 1 down-regulated (PPARG) (Figure3.).

\section{Hub gene validation}

To further investigate the 13 candidate hub genes, we validated their expression in the TCGA_BRCA dataset. The expression levels of all these 13 hub genes were consistent with the results of GEO profiles analysis (Figure4.).

\section{Survival analysis of the identified hub genes}

The KM plot shows that all of the hub genes have a significant difference between high and low expression levels, except FN1 (Figure5.). This result, which is reliable in bioinformatics analyses, indicates that the 12 hub genes have prognostic significance for $\mathrm{BC}$ patients.

\section{Hub gene GSEA analysis}

From the GSEA, we could know that these hub genes are highly related to the "KEGG_CELL_CYCLE", and "HALLMARK_P53_PATHWAY" gene set (Figure6.). The result of GSEA indicates a significant difference $(F D R \leq 0.05)$ in the enrichment of MSigDB Collection and is consistent with GO and KEGG enrichment.

\section{Discussion}

AURKA, also known as aurora kinases, belongs to serine/threonine kinases which share a highly conserved catalytic domain containing auto phosphorylating sites [16]. It positively regulates cell cycle progression and plays a role in the cell centrosome and the spindle microtubules during mitosis [17], which implied that AURKA maybe promotes cancer cell division. On the other hand, it has been widely reported that AURKA is an oncogene to promote tumorigenesis in multiple types of cancer including solid 
tumors and hematological malignancies [16], such as bladder cancer [18, 19], prostate cancer [20, 21], colon cancer [22].

CCNB1 is a regulatory protein involved in mitosis and a critical cell cycle regulator of the G2/M checkpoint [23]. Previous studies have reported that CCNB1 could participate in oncogene pathways among many kinds of cancers, BC, colorectal cancer [24-27]. And initial stage of cancer, it was more recognized by the T cells $[11,28]$. In this study, we found CCNB1 was up-regulated in BC and associated with poor survival.

BUB1B, also called mitotic checkpoint serine/threonine-protein kinase BUB1 beta, is an important element of the mitotic checkpoint [29]. It plays a potential role in spindle checkpoint function in many kinds of cancer [30]. It has reported that inhibited BUB1B leading to cell death, and grow slowly in BC cell [31], consistent with our study.

PRC1(Polycomb Repressive Complexes 1), PRC2 could form a multicomponent complex with PRC2, and these complexes play many roles in the biological process, such as pluripotency, development, and carcinogenesis et al. The abnormal regulation of PRC1 had contributed to cancer progress [32, 33], also some research has confirmed that in prostate cancer and breast cancer $[34,35]$. But the mechanism of PRC1 in cancer still unclear.

Targeting protein for Xenopus kinesin-like protein 2 (TPX2) is a microtubule-associated protein. The expression of TPX2 is strictly regulated by the cell cycle. In general, TPX2 exists in the G1 and S phases of the cell cycle and disappears at the end of cytokinesis [36]. A growing number of papers reported that the high expression of TPX2 was connected with bad and shorter overall survival of patients in many tumors [37-39], but the mechanism of TPX2 in BC is still unknown.

NUSAP1 (encodes nucleolar and spindle-associated protein 1), a nucleolar spindle-associated protein, has been reported that play a complicated role in cell division and mitotic progression, spindle formation, and stability [40]. It inhibits GBM cell growth both in vitro and vivo [41]. NUSAP1 was highly expressed in kinds of malignancies and correlated with poor prognosis in aggressive triple-negative BC [42].

Kinesin family member 20A (KIF20A) is believed to modulate microtubule dynamics [48], which could promote the tumorigenesis and progression of prostate cancer and glioma [49], particularly its biochemical recurrence and metastasis $[48,50]$. Silencing KIF20A could induce prostate cancer cells to death [36]. The aberrantly activated Gli2-KIF20A axis is crucial for the growth of hepatocellular carcinoma and predicts poor prognosis in hepatocellular carcinoma [51].

MCAK(also known as KiF2C), a member of the motor protein-13 motor family, has been reported to undergo large conformational changes when it is converted from solution to microtubule-binding during its catalytic cycle [52]. The activity of MCAK is inhibited by Aurora B kinase through phosphorylation on multiple amino acids within its $\mathrm{N}$-terminus $[53,54]$. Previous studies reported that the high expression of 
KIF2C could serve as an independent marker of poor prognosis in several tumors, including glioma, colorectal cancer, and gastric cancer [55-57], but the roles in the BC reported less.

Ribonucleotide reductase M2 subunit (RRM2), a rate-limiting enzyme involved in DNA synthesis and damage repair, plays important roles in many cellular processes such as cell growth, invasiveness, migration and senescence et al [58]. RRM2 as a tumor driver is frequently overexpressed in various malignancies [59-61]. Others found that the expression level of RRM2 was correlated with invasion, cell differentiation, and metastasis in colorectal carcinoma [62]. Silencing RRM2 attenuated melanoma growth, which was consistent with the maintenance of senescence-associated cell-cycle arrest [63]. Also, it was correlated with lung cancer grade level [64].

Abnormal spindle-like microcephaly associated gene (ASPM), encodes a protein of 3477 amino acids with an NH2-terminal microtubule-binding domain, two calponin homology domains [65]. The function of APSM evidence has pointed out that it is an oncogene and the prognosis has been investigated in various cancers, such as epithelial ovarian cancer, gliomas, pancreatic and prostate cancer, and liver cancer, as well as BC, which devote to tumor progression and involved in poor prognosis [66-71].

Peroxisome proliferator-activated receptor gamma (PPARG) could induce cell cycle arrest, terminal differential, and anti-flammatory [72, 73], and induce G2/M cell cycle arrest by activating P38 in renal cancer and bladder cancer $[74,75]$. Furthermore, PPARG induced down-regulated Wnt/beta-catenin pathway were observed and aberrant in many cancers [76], but sometimes PPARG is promoted in some tumors [72].

\section{Conclusions}

In conclusion, our study identified 13 hub genes that might be involved in the progression of BC with multiple gene expression data sets and a series of comprehensive analyses of bioinformatics. Among those hub genes, 12 targets might be regarded as potential prognostic biomarkers, including AURKA, CCNB1, BUB1B, PRC1, TPX2, NUSAP1, TOP2A, KIF20A, KIF2C, RRM2, ASPM, PPARG. We hope that this study would provide some evidence for the clinical gene-targeting therapy, although somewhere still need more experiment to prove.

\section{Declarations}

\section{Acknowledgements}

Not applicable.

\section{Author contributions}

GC, MY, and JC conceived, designed and wrote the manuscript; YC and HZ analyzed the data; GQ and YD revised the manuscript; GC and MY contributed equally to this study; all authors read and approved the 
final manuscript.

\section{Founding}

This work was supported by Shandong Medical and Health Science and Technology Development Project (Grant No. 202004081034).

\section{Availability of data and materials}

The data sets analyzed during the current study are available from the GEO official websites (https://www.ncbi.nlm.nih.gov/geo/).

\section{Ethics approval and consent to participate}

Not applicable.

\section{Consent for publication}

Not applicable.

\section{Competing interests}

The authors declare that there are no conflict of interest.

\section{References}

1. Cheng, L., et al., Cell division cycle proteinising prognostic biomarker of breast cancer. Biosci Rep, 2020. 40(5).

2. Niu, T., W. Zhang, and W. Xiao, MicroRNA regulation of cancer stem cells in the pathogenesis of breast cancer. Cancer Cell Int, 2021. 21(1): p. 31.

3. Liang, Y., et al., Metastatic heterogeneity of breast cancer: Molecular mechanism and potential therapeutic targets. Seminars in Cancer Biology, 2020. 60: p. 14-27.

4. Liu, F., et al., Identification of core genes and potential molecular mechanisms in breast cancer using bioinformatics analysis. Pathol Res Pract, 2019. 215(7): p. 152436.

5. Wei, L., et al., TCGA-assembler 2: software pipeline for retrieval and processing of TCGA/CPTAC data. Bioinformatics, 2018. 34(9): p. 1615-1617.

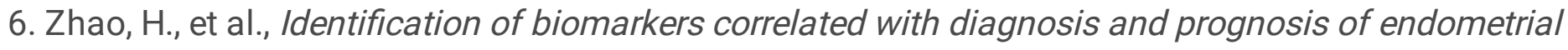
cancer using bioinformatics analysis. Journal of Cellular Biochemistry, 2020. 121(12): p. 4908-4921.

7. The Gene Ontology (GO) project in 2006. Nucleic Acids Res, 2006. 34(Database issue): p. D322-6.

8. Kanehisa, M. and S. Goto, KEGG: kyoto encyclopedia of genes and genomes. Nucleic Acids Res, 2000. 28(1): p. 27-30. 
9. Wang, X., et al., Modularity analysis based on predicted protein-protein interactions provides new insights into pathogenicity and cellular process of Escherichia coli 0157:H7. Theor Biol Med Model, 2011. 8: p. 47.

10. Song, W., et al., Analysis of Circular RNA-Related Competing Endogenous RNA Identifies the ImmuneRelated Risk Signature for Colorectal Cancer. Front Genet, 2020. 11: p. 505.

11. Liu, S., et al., Identification of candidate biomarkers correlated with the pathogenesis and prognosis of breast cancer via integrated bioinformatics analysis. Medicine (Baltimore), 2020. 99(49): p. e23153.

12. Cai, Y., et al., Identification of five hub genes as monitoring biomarkers for breast cancer metastasis in silico. Hereditas, 2019. 156: p. 20.

13. Baade, P., Geographical Variation in Breast Cancer Outcomes. Int J Environ Res Public Health, 2017. 14(5).

14. Liang, Y., et al., Metastatic heterogeneity of breast cancer: Molecular mechanism and potential therapeutic targets. Semin Cancer Biol, 2020. 60: p. 14-27.

15. Siegel, R.L., K.D. Miller, and A. Jemal, Cancer statistics, 2019. CA Cancer J Clin, 2019. 69(1): p. 7-34.

16. Du, R., et al., Targeting AURKA in Cancer: molecular mechanisms and opportunities for Cancer therapy. Mol Cancer, 2021. 20(1): p. 15.

17. Carvalhal, S., et al., The nucleoporin ALADIN regulates Aurora A localization to ensure robust mitotic spindle formation. Mol Biol Cell, 2015. 26(19): p. 3424-38.

18. de Martino, M., et al., Aurora A Kinase as a diagnostic urinary marker for urothelial bladder cancer. World J Urol, 2015. 33(1): p. 105-10.

19. Lei, Y., et al., Prognostic significance of Aurora-A expression in human bladder cancer. Acta Histochem, 2011. 113(5): p. 514-8.

20. Matarasso, N., et al., Functional analysis of the Aurora Kinase A lle31 allelic variant in human prostate. Neoplasia, 2007. 9(9): p. 707-15.

21. Buschhorn, H.M., et al., Aurora-A over-expression in high-grade PIN lesions and prostate cancer. Prostate, 2005. 64(4): p. 341-6.

22. Lentini, L., et al., Aurora-A transcriptional silencing and vincristine treatment show a synergistic effect in human tumor cells. Oncol Res, 2008. 17(3): p. 115-25.

23. Liu, J., et al., VPS33B modulates c-Myc/p53/miR-192-3p to target CCNB1 suppressing the growth of non-small cell lung cancer. Molecular Therapy - Nucleic Acids, 2021. 23: p. 324-335.

24. Fei, F., et al., The subcellular location of cyclin B1 and CDC25 associated with the formation of polyploid giant cancer cells and their clinicopathological significance. Lab Invest, 2019. 99(4): p. 483-498.

25. Wang, L., et al., Sanyang Xuedai enhances the radiosensitivity of human non-small cell lung cancer cells via increasing iNOS/NO production. Biomed Pharmacother, 2018. 102: p. 618-625. 
26. Monteiro, L.S., et al., Prognostic Significance of Cyclins A2, B1, D1, and E1 and CCND1 Numerical Aberrations in Oral Squamous Cell Carcinomas. Anal Cell Pathol (Amst), 2018. 2018: p. 7253510.

27. Wang, W., et al., The p53/miR-193a/EGFR feedback loop function as a driving force for non-small cell lung carcinoma tumorigenesis. Ther Adv Med Oncol, 2019. 11: p. 1758835919850665.

28. Egloff, A.M., et al., Evaluation of anticyclin B1 serum antibody as a diagnostic and prognostic biomarker for lung cancer. Ann N Y Acad Sci, 2005. 1062: p. 29-40.

29. Sackton, K.L., et al., Synergistic blockade of mitotic exit by two chemical inhibitors of the APC/C. Nature, 2014. 514(7524): p. 646-9.

30. Liu, S., et al., Identification of candidate biomarkers correlated with the pathogenesis and prognosis of breast cancer via integrated bioinformatics analysis. Medicine, 2020. 99(49): p. e23153.

31. Koyuncu, D., et al., Spindle assembly checkpoint gene BUB1B is essential in breast cancer cell survival. Breast Cancer Res Treat, 2021. 185(2): p. 331-341.

32. Brynychova, V., et al., Genetic and functional analyses do not explain the association of high PRC1 expression with poor survival of breast carcinoma patients. Biomed Pharmacother, 2016. 83: p. 857864.

33. Zhang, B., et al., Elevated PRC1 in gastric carcinoma exerts oncogenic function and is targeted by piperlongumine in a p53-dependent manner. J Cell Mol Med, 2017. 21(7): p. 1329-1341.

34. Luo, H.W., et al., Protein regulator of cytokinesis 1 overexpression predicts biochemical recurrence in men with prostate cancer. Biomed Pharmacother, 2016. 78: p. 116-120.

35. Yun, H.J., et al., Transcriptional targeting of gene expression in breast cancer by the promoters of protein regulator of cytokinesis 1 and ribonuclease reductase 2. Exp Mol Med, 2008. 40(3): p. 345-53.

36. Heidebrecht, H.J., et al., p100: a novel proliferation-associated nuclear protein specifically restricted to cell cycle phases S, G2, and M. Blood, 1997. 90(1): p. 226-33.

37. Hsu, C.W., et al., Targeting TPX2 Suppresses the Tumorigenesis of Hepatocellular Carcinoma Cells Resulting in Arrested Mitotic Phase Progression and Increased Genomic Instability. J Cancer, 2017. 8(8): p. 1378-1394.

38. Liang, B., et al., TPX2 Level Correlates with Hepatocellular Carcinoma Cell Proliferation, Apoptosis, and EMT. Dig Dis Sci, 2015. 60(8): p. 2360-72.

39. Liu, H.C., et al., Upregulation of the TPX2 gene is associated with enhanced tumor malignance of esophageal squamous cell carcinoma. Biomed Pharmacother, 2013. 67(8): p. 751-5.

40. Mills, C.A., et al., Nucleolar and spindle-associated protein 1 (NUSAP1) interacts with a SUMO E3 ligase complex during chromosome segregation. J Biol Chem, 2017. 292(42): p. 17178-17189.

41. Qian, Z., et al., Prognostic value of NUSAP1 in progression and expansion of glioblastoma multiforme. J Neurooncol, 2018. 140(2): p. 199-208.

42. Chen, L., et al., High Levels of Nucleolar Spindle-Associated Protein and Reduced Levels of BRCA1 Expression Predict Poor Prognosis in Triple-Negative Breast Cancer. PLoS One, 2015. 10(10): p. e0140572. 
43. Tang, Y., Y. Zhang, and X. Hu, Identification of Potential Hub Genes Related to Diagnosis and Prognosis of Hepatitis B Virus-Related Hepatocellular Carcinoma via Integrated Bioinformatics Analysis. Biomed Res Int, 2020. 2020: p. 4251761.

44. Deng, J.L., Y.H. Xu, and G. Wang, Identification of Potential Crucial Genes and Key Pathways in Breast Cancer Using Bioinformatic Analysis. Front Genet, 2019. 10: p. 695.

45. Zhang, R., et al., Proliferation and invasion of colon cancer cells are suppressed by knockdown of TOP2A. J Cell Biochem, 2018. 119(9): p. 7256-7263.

46. Liu, H.Q., S.L. Zhang, and S. Song, HER-2/neu and TOPlla expression in gastric cancer reflect disease severity. Hepatogastroenterology, 2012. 59(116): p. 1290-3.

47. Shen, J., et al., Identification of key biomarkers associated with development and prognosis in patients with ovarian carcinoma: evidence from bioinformatic analysis. J Ovarian Res, 2019. 12(1): p. 110.

48. $\mathrm{Yu}, \mathrm{H}$., et al., FOXM1 modulates docetaxel resistance in prostate cancer by regulating KIF20A. Cancer Cell Int, 2020. 20(1): p. 545.

49. He, Q., et al., Long non-coding RNA UCA1 upregulates KIF20A expression to promote cell proliferation and invasion via sponging miR-204 in cervical cancer. Cell Cycle, 2020. 19(19): p. 2486-2495.

50. Song, Z., et al., The Identification of Potential Biomarkers and Biological Pathways in Prostate Cancer. J Cancer, 2019. 10(6): p. 1398-1408.

51. Shi, C., et al., Aberrantly activated Gli2-KIF20A axis is crucial for growth of hepatocellular carcinoma and predicts poor prognosis. Oncotarget, 2016. 7(18): p. 26206-19.

52. McHugh, T., et al., The depolymerase activity of MCAK shows a graded response to Aurora $B$ kinase phosphorylation through allosteric regulation. J Cell Sci, 2019. 132(4).

53. Andrews, P.D., et al., Aurora B regulates MCAK at the mitotic centromere. Dev Cell, 2004. 6(2): p. 25368.

54. Lan, W., et al., Aurora B phosphorylates centromeric MCAK and regulates its localization and microtubule depolymerization activity. Curr Biol, 2004. 14(4): p. 273-86.

55. Bie, L., et al., Kinesin family member $2 C$ (KIF2C/MCAK) is a novel marker for prognosis in human gliomas. Clin Neurol Neurosurg, 2012. 114(4): p. 356-60.

56. Ishikawa, K., et al., Mitotic centromere-associated kinesin is a novel marker for prognosis and lymph node metastasis in colorectal cancer. Br J Cancer, 2008. 98(11): p. 1824-9.

57. Nakamura, Y., et al., Clinicopathological and biological significance of mitotic centromere-associated kinesin overexpression in human gastric cancer. Br J Cancer, 2007. 97(4): p. 543-9.

58. Nordlund, P. and P. Reichard, Ribonucleotide reductases. Annu Rev Biochem, 2006. 75: p. 681-706.

59. Jin, C.Y., et al., High expression of RRM2 as an independent predictive factor of poor prognosis in patients with lung adenocarcinoma. Aging (Albany NY), 2020. 12.

60. Grolmusz, V.K., et al., Cell cycle dependent RRM2 may serve as proliferation marker and pharmaceutical target in adrenocortical cancer. Am J Cancer Res, 2016. 6(9): p. 2041-2053. 
61. Grossi, F., et al., Expression of Ribonucleotide Reductase Subunit-2 and Thymidylate Synthase Correlates with Poor Prognosis in Patients with Resected Stages I-III Non-Small Cell Lung Cancer. Dis Markers, 2015. 2015: p. 302649.

62. Lu, A.G., et al., Emerging roles of the ribonucleotide reductase M2 in colorectal cancer and ultravioletinduced DNA damage repair. World J Gastroenterol, 2012. 18(34): p. 4704-13.

63. Fatkhutdinov, N., et al., Targeting RRM2 and Mutant BRAF Is a Novel Combinatorial Strategy for Melanoma. Mol Cancer Res, 2016. 14(9): p. 767-75.

64. Hsu, N.Y., et al., Expression status of ribonucleotide reductase small subunits hRRM2/p53R2 as prognostic biomarkers in stage I and II non-small cell lung cancer. Anticancer Res, 2011. 31(10): p. 3475-81.

65. Gao, Z.Y., et al., ASPM predicts poor prognosis and regulates cell proliferation in bladder cancer. Kaohsiung J Med Sci, 2020. 36(12): p. 1021-1029.

66. Lin, S.Y., et al., ASPM is a novel marker for vascular invasion, early recurrence, and poor prognosis of hepatocellular carcinoma. Clin Cancer Res, 2008. 14(15): p. 4814-20.

67. Alsiary, R., et al., Deregulation of microcephalin and ASPM expression are correlated with epithelial ovarian cancer progression. PLoS One, 2014. 9(5): p. e97059.

68. Bikeye, S.N., et al., ASPM-associated stem cell proliferation is involved in malignant progression of gliomas and constitutes an attractive therapeutic target. Cancer Cell Int, 2010. 10: p. 1.

69. Xie, J.J., et al., High expression of ASPM correlates with tumor progression and predicts poor outcome in patients with prostate cancer. Int Urol Nephrol, 2017. 49(5): p. 817-823.

70. Turnbull, A.K., et al., Accurate Prediction and Validation of Response to Endocrine Therapy in Breast Cancer. J Clin Oncol, 2015. 33(20): p. 2270-8.

71. Wang, W.Y., et al., A gene expression signature of epithelial tubulogenesis and a role for ASPM in pancreatic tumor progression. Gastroenterology, 2013. 145(5): p. 1110-20.

72. Huang, R., et al., The Role of Peroxisome Proliferator-Activated Receptors (PPARs) in Pan-Cancer. PPAR Res, 2020. 2020: p. 6527564.

73. Bandera Merchan, B., F.J. Tinahones, and M. Macias-Gonzalez, Commonalities in the Association between PPARG and Vitamin D Related with Obesity and Carcinogenesis. PPAR Res, 2016. 2016: p. 2308249.

74. Fujita, M., et al., Cytotoxicity of troglitazone through PPARgamma-independent pathway and p38 MAPK pathway in renal cell carcinoma. Cancer Lett, 2011. 312(2): p. 219-27.

75. Plissonnier, M.L., et al., Insights on distinct pathways of thiazolidinediones (PPARgamma ligand)promoted apoptosis in TRAIL-sensitive or -resistant malignant urothelial cells. Int J Cancer, 2010. 127(8): p. 1769-84.

76. Vallee, A., Y. Lecarpentier, and J.N. Vallee, Curcumin: a therapeutic strategy in cancers by inhibiting the canonical WNT/beta-catenin pathway. J Exp Clin Cancer Res, 2019. 38(1): p. 323. 
Figures
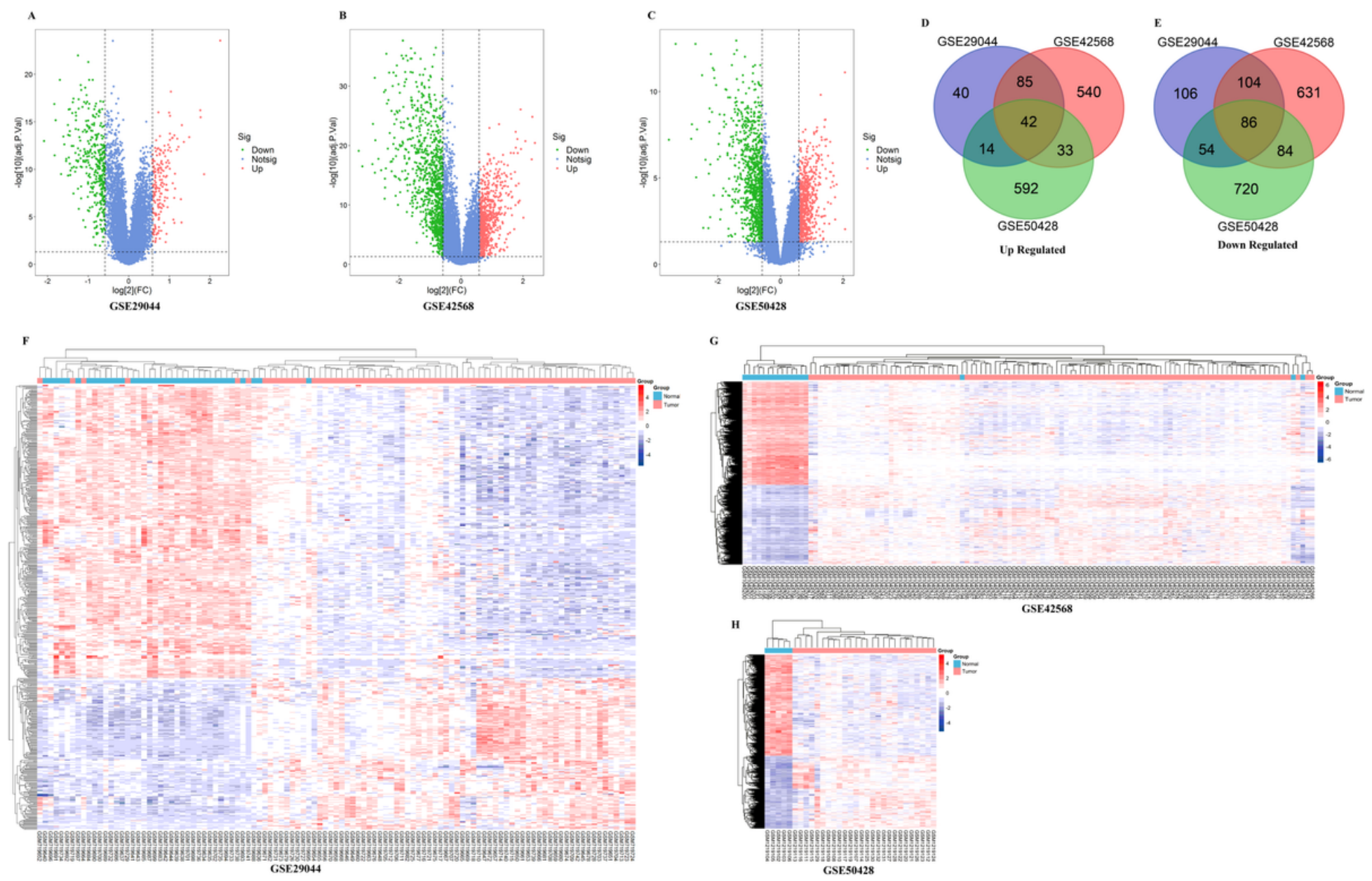

Figure 1

DEGs of three GEO profiles (A-C, The volcano map of DEGs three GEO profile. F-H, The heat map of DEGs three GEO profile. D, E, Venn diagram of three GEO profiles DEGs.)

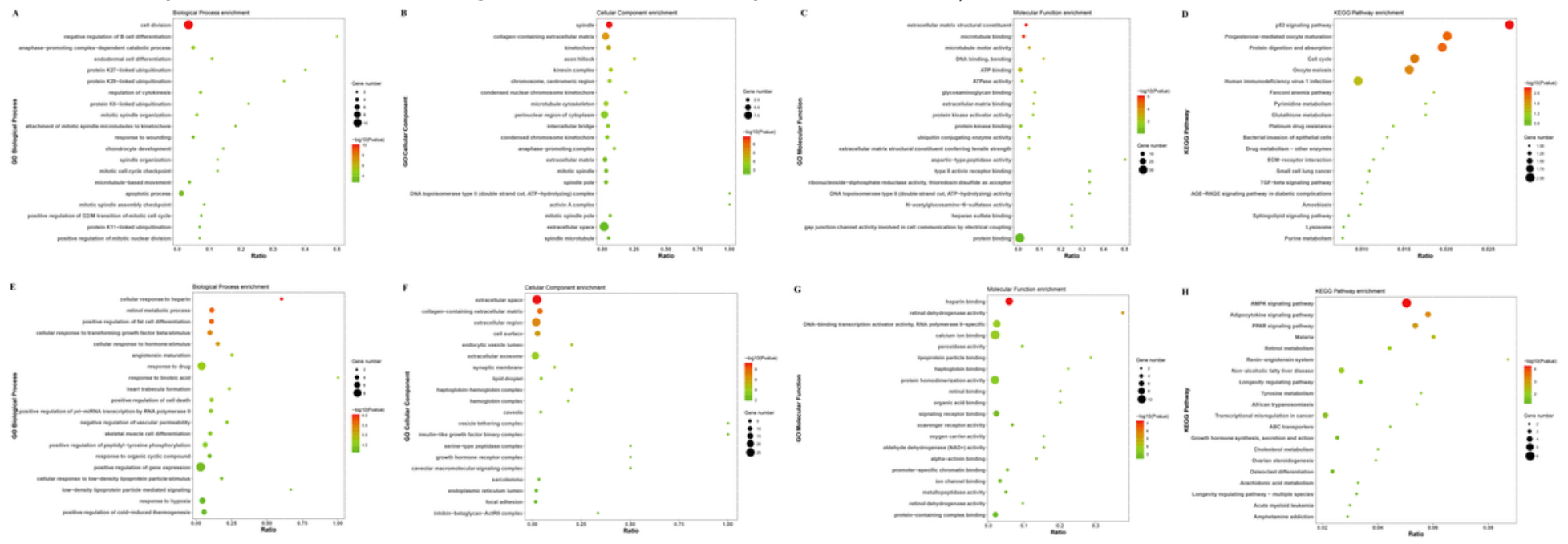

Figure 2 
GO function and KEGG pathway analysis of DEGs. (A-D, GO function, and KEGG pathway analysis of upregulated DEGs. E-H GO function and KEGG pathway analysis of down-regulated DEGs.)

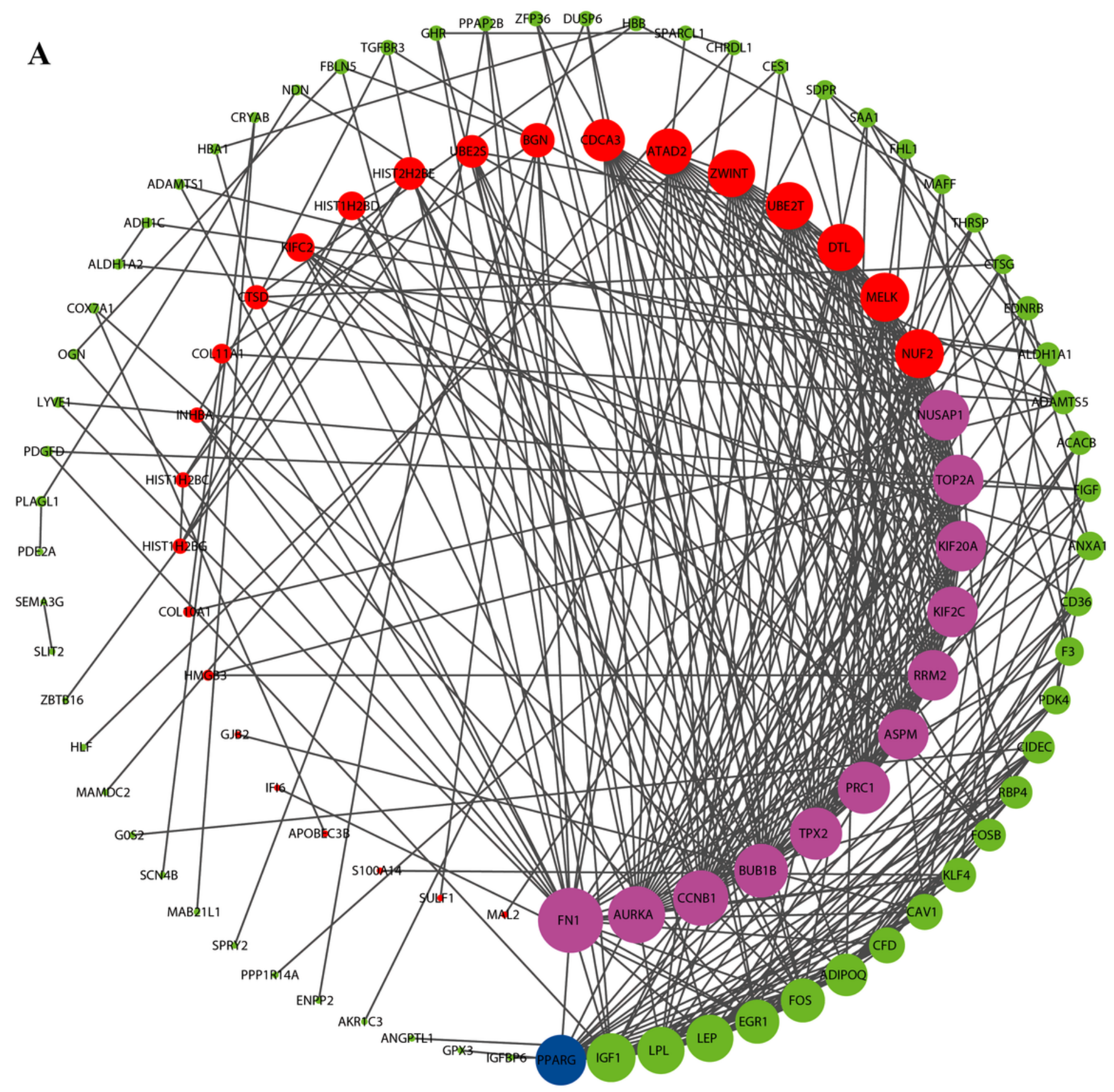

Figure 3

PPI analysis of hub genes of DEGs. (A, Red color is up-regulated DEGs, purple is up-regulated hub genes, green is down-regulated genes, blue is down-regulated hub genes.) 

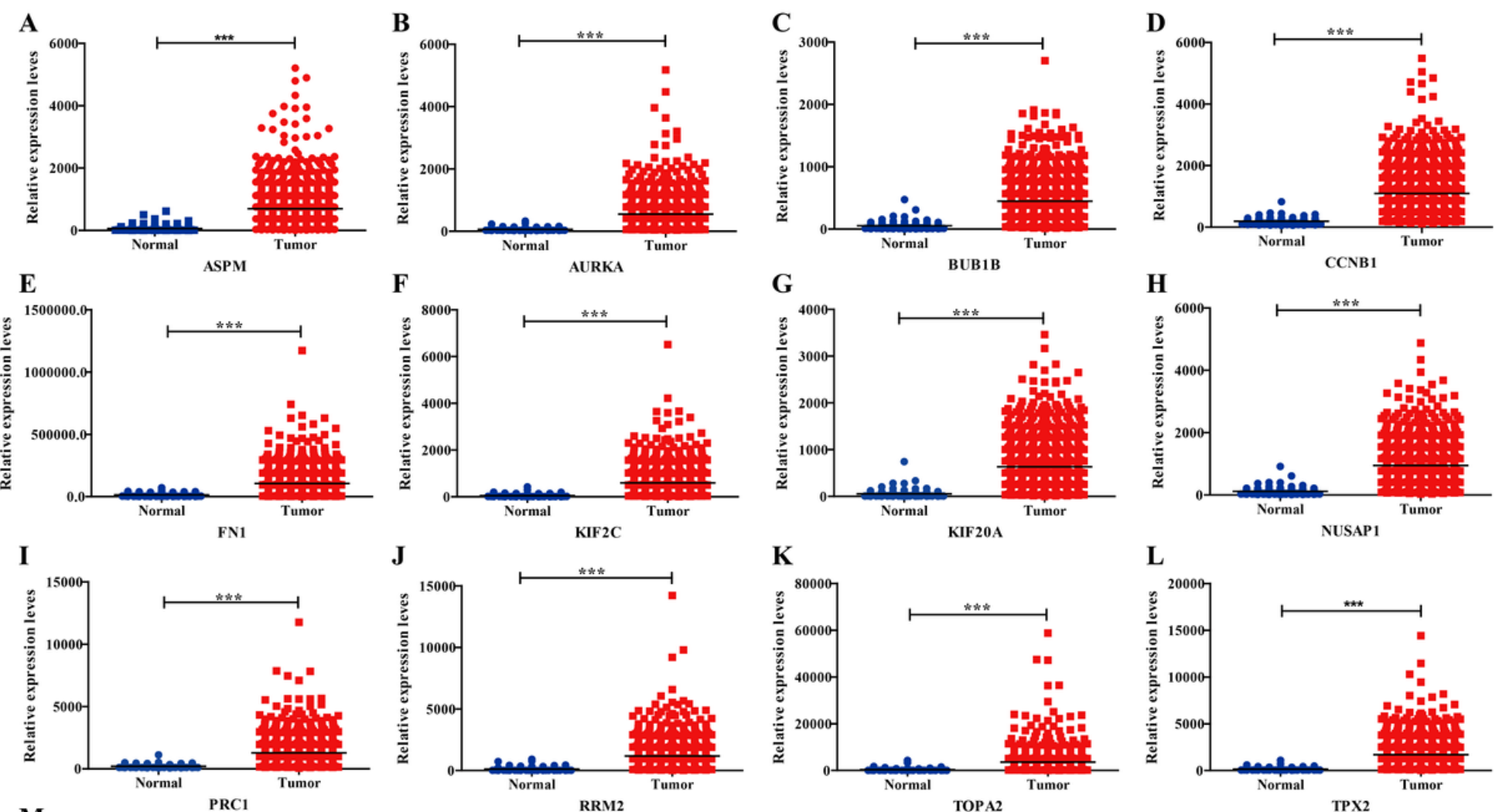

$\mathbf{K}$
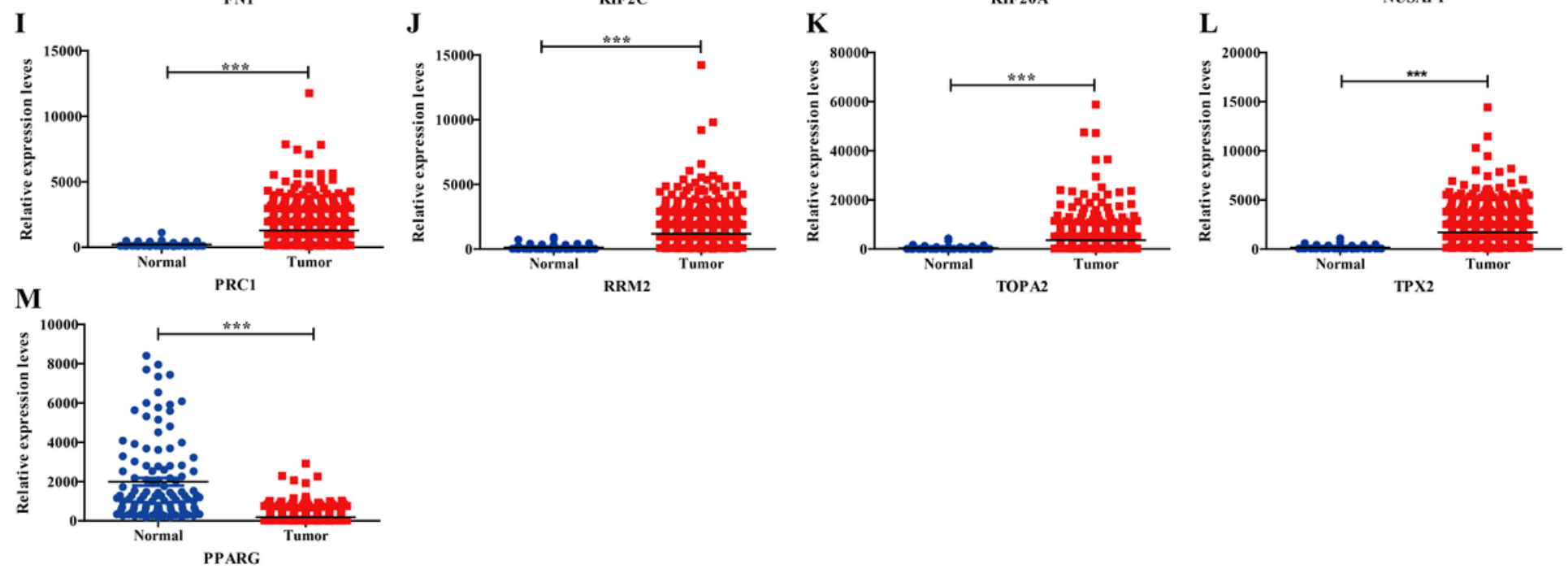

Figure 4

Expression validation of 13 hub targets in BC compared with adjacent tissues from TCGA data sets. (A-L, Up-regulated hub genes of BC from TCGA database. $M$, Down-regulated hub genes of BC from TCGA database.) 

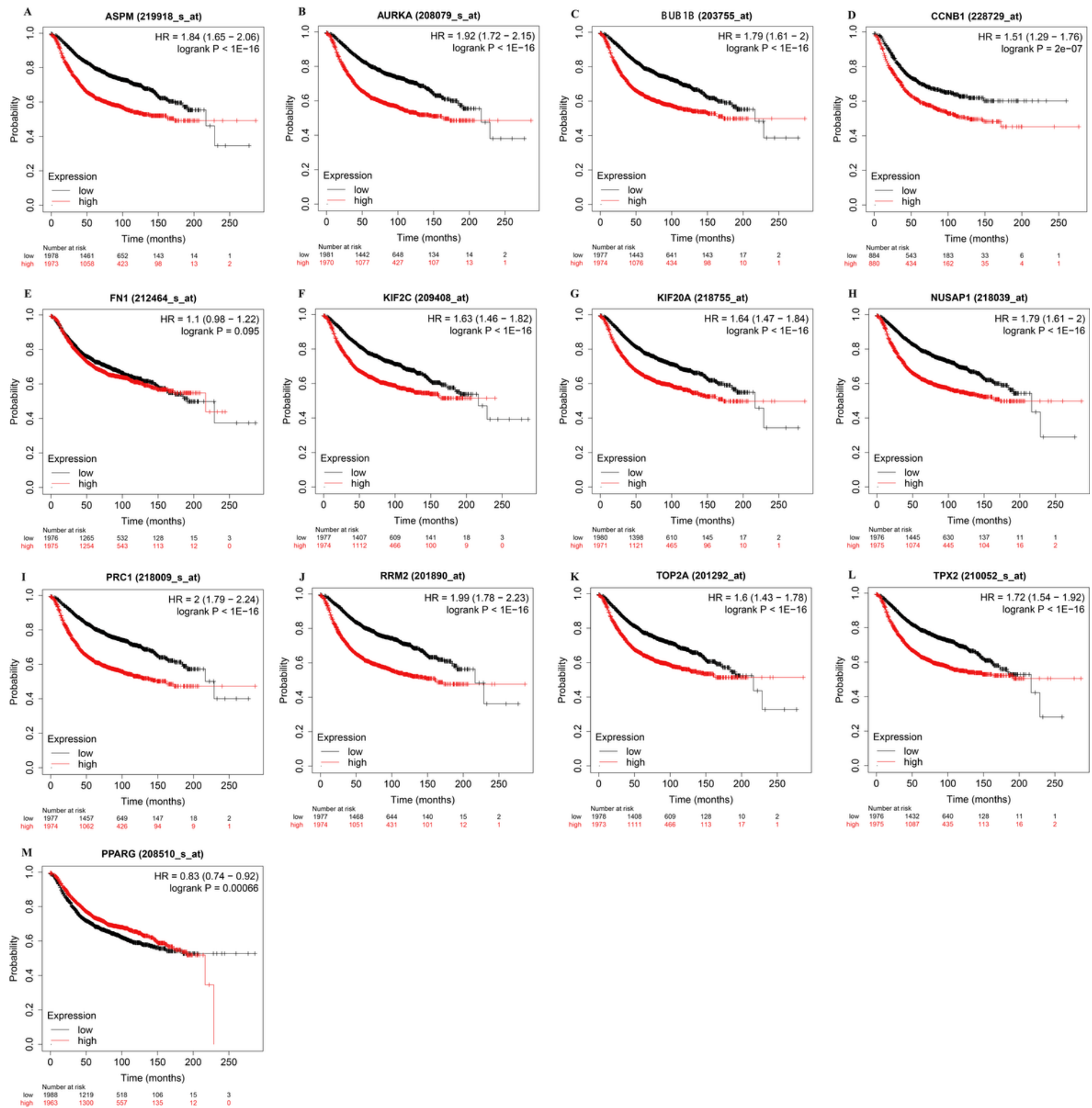

Figure 5

The prognostic gene signature of hub genes in the BC patients. (A-L, Overall survival of up-regulated hub genes. $M$, Overall survival of down-regulated hub genes.) 

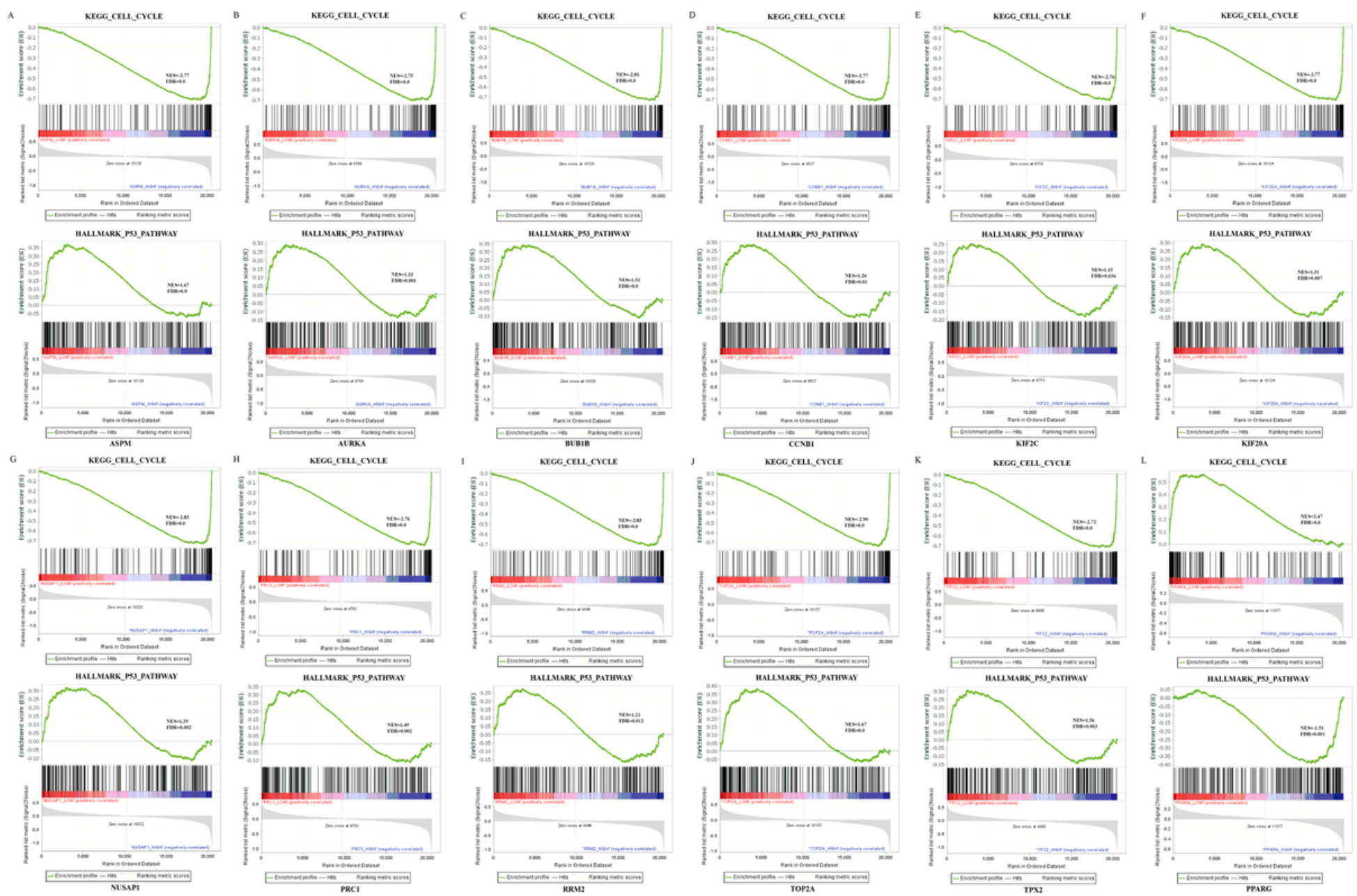

Figure 6

Enrichment plots from GSEA. (A-K, Enrichment plots from GSEA of up-regulated hub genes. Enrichment plots from GSEA of down-regulated hub genes.) 